УДК. 663.14. 033, 663.143.2

\title{
ЭКСПЕРИМЕНТАЛЬНОЕ ОПРЕДЕЛЕНИЕ КОЭФФИЦИЕНТА МАССООБМЕНА ПРИ КУЛЬТИВИРОВАНИИ МИКРООРГАНИЗМОВ С ПРИМЕНЕНИЕМ МЕТОДА ДИСКРЕТНО-ИМПУЛЬСНОГО ВВОДА ЭНЕРГИИ (ДИВЭ)
}

Ободович А.Н. ${ }^{1}$, докт. техн. наук, Мудрак Т.Е. ${ }^{2}$, канд. техн. наук, Костик С.И. ${ }^{1}$, канд. техн. наук, Сидоренко В.В. ${ }^{1}$

${ }^{1}$ Институт технической теплофизики НАН Украины, ул. Желябова, 2а, Киев, 03680, Украина

${ }^{2}$ Национальный университет пищевых технологий МОН Украины, ул. Владимирская, 68, Киев-33, 01601, Украина

У статті висвітлено шляхи інтенсифікації процесу культивування мікроорганізмів, а також запропоновано відповідне тепломасообмінне обладнання. Представлена методика визначення коефіцієнта масообміну на прикладі культивування хлібопекарських дріжджів.
В статье освещены пути интенсификации процесса культивирования микроорганизмов, а также предложено соответствующее тепломассообменное оборудование. Представлена методика определения коэффициента массообмена на примере культивирования хлебопекарских дрожжей.
The article highlights the ways of intensifying the process of cultivation of microorganisms, as well as to propose appropriate heat and mass transfer equipment. The technique of determining the mass-transfer coefficient on the example of the culture bakery yeast.

Библ. 5, рис. 1.

Ключевые слова: аэрация, коэффициент массообмена, роторно- пульсационный аппарат, дрожжи.

Скорость биохимических процессов, протекающих в диффузионной области, определяется интенсивностью тепло- и массообмена. Введение внешней энергии в среду интенсифицирует указанные процессы.

При вводе энергии с помощью механического перемешивания среды путем использования центробежных сил, механических вибраций, ультразвукового воздействия и т. п., зоны максимальной энергии по объему аппарата распределяются неравномерно. Интенсивное турбулентное обтекание дисперсной фазы, ее дробление и высокий уровень процессов тепломассообмена обеспечивается не во всем объеме аппарата, а лишь в локальной зоне ввода энергии. В остальной части рабочего объема аппарата скорость обтекания дисперсных частиц невелика и поэтому процессы тепломассообмена недостаточно интенсивны.

Значительная часть энергии расходуется на перемешивание с целью ввода дисперсных частиц и жидкости в локальную зону подвода энергии, что снижает КПД аппарата. Поэтому традиционные методы локального ввода энергии связаны с большими энергозатратами и необходимостью длительного проведения процесса, поскольку относительный объем локальной зоны, как правило, очень мал. Именно это препятствует зачастую возможности непрерывного ведения процесса, определяет высокую металлоемкость аппаратов, их низкую экономическую эффективность.

В Институте технической теплофизики НАНУ, в течение ряда лет разрабатывается принципиально новое направление - интенсификация тепломассообменных процессов в гетерогенных системах методом дискретно-импульсного ввода энергии (ДИВЭ) [1]. Суть метода заключается в том, что энергия, вводимая в аппарат для интенсификации тепломассообмеиных процессов, распределяется дискретно во времени и по объему дисперсной системы в рабочей зоне аппарата. При этом к поверхности раздела фаз между каждой дисперсной частицей и окружающей средой подводится необходимое количество энергии. 
В данной работе метод ДИВЭ применяли для интенсификации растворения и массопереноса кислорода и питательных веществ из культуральной среды в клетку микроорганизма.

Исследование массообмена и аэрации среды с применением метода ДИВЭ проводили на примере культивирования дрожжей расы Saharomices cerevisae, используемых для сбраживания углеводов.
С целью интесификации процесса культивирования микроорганизмов был создан тепломассообменный аппарат (ферментационная установка с дискретно-импульсным вводом энергии), основным рабочим органом которого является роторно-пульсационный аппарат (РПА), в котором реализуется метод ДИВЭ (рис. 1).

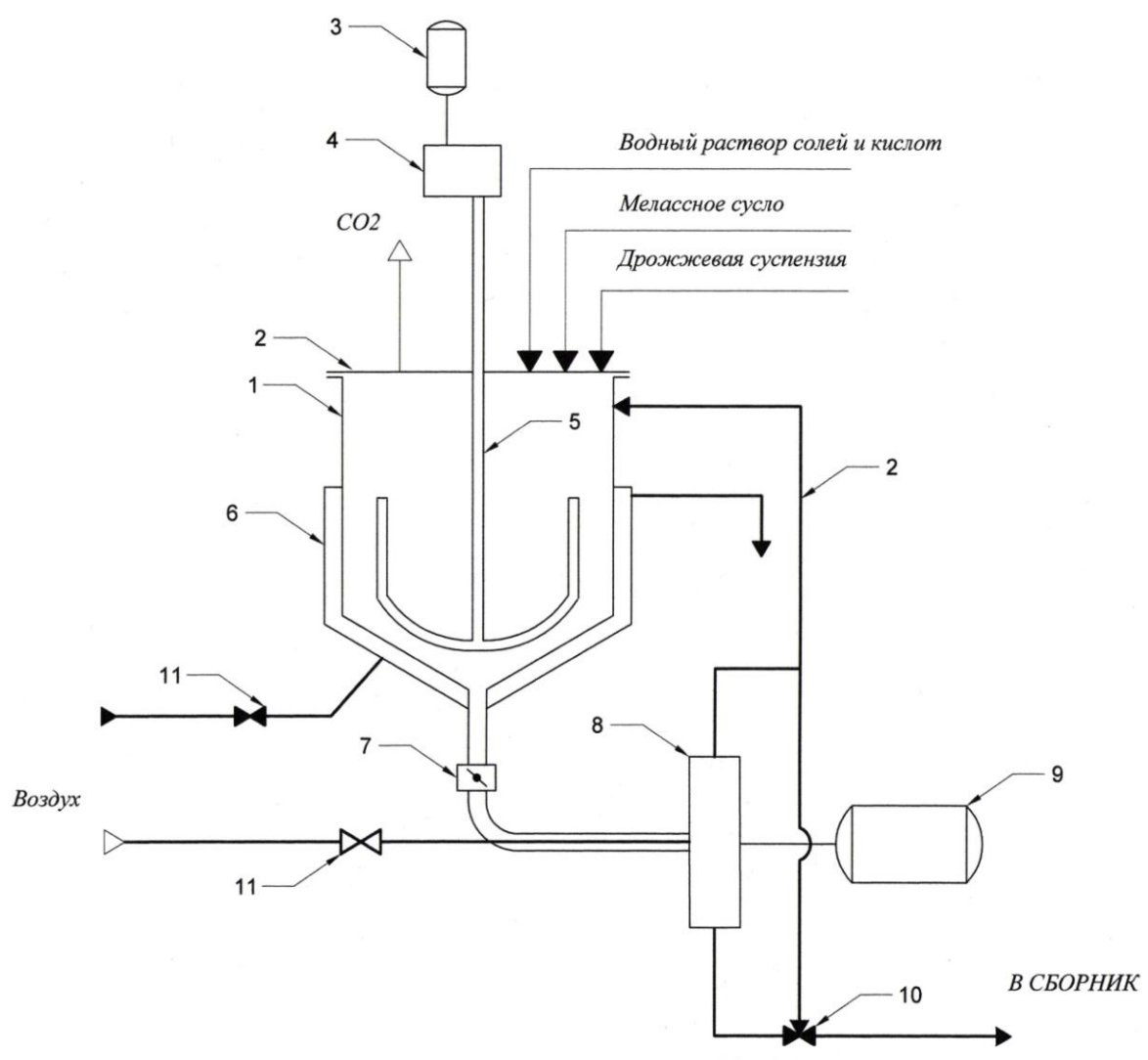

Рис. 1. Аппаратурно-технологическая схема тепломассообменной установки для культивирования микроорганизмов (на примере культивирования хлебопекарских дрожсжей).

1 - бункер; 2 - крышка бункера; 3 - двигатель мешалки; 4 -редуктор; 5 -мешалка;

6-рубашка; 7 -заслонка; 8-роторно-пульсационный аппарат; 9 - двигатель;

10 - трёхходовой кран; 11 - двухходовой кран; 12 - трубопровод рециркуляции.

Установка работает следующим образом. В приемный бункер 1 подается мелассное сусло, водный раствор солей и кислот и засевные дрожжи. Включают мешалку 5 и проводят предварительное перемешивание ингридиентов. Затем включают роторно-пульсационный аппарат 8, открывают заслонку 7 и 3-х ходовой кран 10, который находится в положении, при котором культуральная среда циркулирует по контуру приёмный бункер - роторно-пульсационный аппарат - приёмный бункер.

Во время рециркуляции смеси открывают 2-хходовой кран, через который поступает воздух из атмосферы, за счёт разряжения, создаваемого в приёмном патрубке роторно-пульсационного аппарата. Таким образом смесь культуральной среды и дрожжей насыщается воздухом, который нахо- 
дится в газообразном состоянии. Полученная смесь направляется в рабочую камеру роторно-пульсационного аппарата, где подвергается обработке методом дискретно-импульсного ввода энергии.

При попадании в полость рабочих органов РПА происходит диспергирование смеси пузырьков воздуха в питательной среде. Вышедшие из рабочей области пузырьки воздуха имеют наноразмеры, что значительно увеличивает поверхность контакта фаз. Кроме того, смесь воздуха и питательной среды, проходя через РПА, подвергается воздействию высокочастотных пульсаций давления, сильной турбулизации, разрыву сплошности потока, сдвиговых напряжений и т.д.

Роторно - пульсационный аппарат состоит из корпуса с входным и выходным патрубками, системы трубопроводов для рециркуляции или вывода готовой продукции, горизонтального роторного узла, состоящего из двух роторов и одного статора, размещающихся в одном неподвижном стакане. На цилиндрических поверхностях статора и роторов выполнены 64 сквозные продольные прорези. Радиальный зазор между рабочими органами ротор - статор - ротор составляет 150 ...300 мкм. Во время вращения ротора с частотой $1000 . .5000$ мин $^{-1}$ по очереди происходит совмещение пазов статора и роторов, что является причиной возникновения значительных перепадов давления, высокоградиентных течений в зазорах, а также высоких градиентов сдвиговых напряжений. Локальные скорости сдвига потока могут изменяться от $100 \cdot 10^{2}$ до $100 \cdot 10^{3} \mathrm{c}^{-1}$, частоты импульсов от 0,1 до 10 кГц.

Благодаря явлениям, происходящих в роторнопульсационном аппарате увеличивается коэффициент массоперерноса, повышается площадь контакта фаз, что способствует увеличению скорости растворения кислорода и быстрой доставки его, а также питательных веществ в дрожжевую клетку. В конечном итоге, это приводит к повышению удельной скорости роста дрожжей $(\mu)$ на $35 . .45 \%$.

При культивировании микроорганизмов в ферментаторе с дискретно-импульсным вводом энергии процесс может проходить в режиме непрерывной рециркуляции за определенное количество циклов, с остановками или в прямотоке за один проход.

Основные процессы абсорбции и массопереноса проходят в зазорах и щелях цилиндров роторно- пульсационного аппарата.

Определить дисперсность пузырьков воздуха, площадь контакта фаз и другие показатели, влияющие на коэффициент массопереноса невозможно. Поэтому нами предложено определять скорость растворения кислорода в питательной среде, применяя уравнение массообмена [2].

$K_{D}=\frac{d c}{d t}=\beta F(C o-C)$,

где $K_{D}-$ скорость абсорбции кислорода, кг $\mathrm{O}_{2} /$ $\mathrm{M}^{3} \cdot$; $C$ - $о$ концентрация кислорода в жидкости при полном насыщении ее, которое может быть достигнуто при данной температуре и парциальном давлении кислорода в продуваемом воздухе, кг $\mathrm{O}_{2} / \mathrm{M}^{3} ; C$ - фактическая концентрация̃ кислорода в культуральной жидкости $\mathrm{O}_{2} / \mathrm{M}^{3}$; $\beta$ - константа диффузии кислорода для данной среды и температуры, м/ч; $F$ - удельная поверхность раздела между жидкой и газовой фазами, $\mathrm{M}^{2} / \mathrm{M}^{3}$

Произведение $\beta F$ характеризует эффективность аэрирующего устройства и обозначается символом $K_{L a}$. Поэтому выражение (1) может быть написано так

$$
K_{D}=\frac{d c}{d t}=K l a(C o-C) .
$$

Величина $K_{L a}$ зависит от качества питательной среды, типа аэрационного устройства и гидродинамического режима работы аппарата, в котором происходит абсорбция кислорода.

Сравнивать эффективность различных аэраторов и аппаратов можно лишь по значению $K_{L a}$ для каждого из них отдельно, а не скорости абсорбции, так как последняя тем больше, чем ближе к нулю фактическое содержание кислорода в среде $(\mathrm{C} \sim 0)$ тем меньше, чем ближе к насыщению содержание в ней кислорода $(\mathrm{Co})$. Следовательно, скорость растворения кислорода, т. е. увеличение его концентрации в единицу времени, уменьшается с увеличением количества кислорода, растворенного в среде.

Насыщение среды кислородом зависит от всех условий, влияющих на поверхность контакта между жидкой и газовой фазами, а также от температуры. В случае аэрации через трубчатые аэраторы $K_{L a}$ зависит от скорости прохождения 
воздуха через отверстия, от уровня заполнения аппарата. Если применяются вращающиеся аэраторы, $K_{L a}$ зависит, соответственно, от числа оборотов мешалки и глубины ее погружения. В эрлифтных аэраторах $K_{L a}$ зависит от размеров направляющего цилиндра (диффузора), интенсивности циркуляции жидкости и других факторов.

При использовании ферментационной установки с дискретно-импульсным вводом энергии $K_{L a}$ будет зависеть от угловой скорости вращения ротора, частоты пульсаций, величины давления, скорости потока в радиальном направлении, величине зазора между статором и ротором, скорости и напряжения сдвига потока и т.Д.

Значение $K_{L a}$ можно определить: 1) сульфитным методом; 2) аэрацией обезкислороженной воды или бражки, что достигается добавлением сернистого раствора, содержащего ионы $\mathrm{S}++$, или путем пропускания через жидкость инертного газа; 3) аэрацией среды в присутствии известного количества дрожжей с заранее установленным коэффициентом скорости использования ими кислорода $(K r)$ [3]. Кислород в этом случае определяют полярографическим методом. Самым достоверным является третий метод, учитывающий все факторы, влияющие на величину $K_{L a}$, пригодный для лабораторных и заводских исследований. При определении величины $K r$ закрывают на время доступ воздуха в среду и фиксируют убыль кислорода, быстро ассимилируемого дрожжами, в зависимости от времени. Когда в среде достаточно дрожжей, величина $C$ за несколько секунд падает до нуля.

Устанавливая $K_{L a}$, следует сохранять постоянную температуру и степень заполнения аппарата, т. е. высоту слоя жидкости, а также избегать добавления пеногасителя, снижающего величину $K_{L a}$.

Растворенный в жидкости кислород потребляется дрожжевыми клетками со скоростью

$$
\frac{d c}{d t}=K r=\mu \cdot x \cdot q
$$

где $K r$ - скорость усвоения кислорода дрожжа-

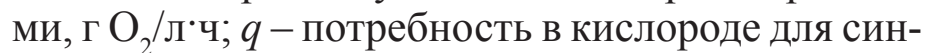
теза АС̆Д, г $\mathrm{O}_{2} / \Gamma ; \mu$ - удельная скорость роста дрожжей $\Gamma / \Gamma^{*} ч$, или $\frac{1}{q} ; x-$ концентрация дрожжей в среде, г/л.
При непрерывно-проточном культивировании, когда популяция клеток находится в состоянии подвижного равновесия, скорости растворения кислорода и потребления его

$$
K_{D}=K r=\mu \cdot x \cdot q=K_{L a}\left(C_{0}-C\right) .
$$

Заменив в уравнении (4) $q$ обратной величиной, т. е. количеством дрожжей $\left(y_{o 2}\right)$ в г, выросших при использовании 1г кислорода, и подставив вместо $\mu$ равное ему значение $D$ получим равенство

$$
K_{D}=\frac{D \cdot x}{y 02} \text {. }
$$

Таким образом для расчета величины $K_{D}$ требуется экспериментально определить величину $\left(y_{o 2}\right)$ в г (или $\left.q\right)$, скорость разбавления $(D)$ и соответствующую ей концентрацию дрожжей в среде.

Пример: $D=0,12$; концентрация дрожжей $x=10$ г/л; $q=1,5$ г $\mathrm{O}_{2} /$ г. При этих условиях величина $\left(y_{o 2}\right)$ составит

$$
\begin{gathered}
y_{o 2}-1: 1,5=0,67, \text { откуда } \\
K_{D}=0,12 \cdot 10 / 0,67=1,8 \text { г/л ч } .
\end{gathered}
$$

Зная скорость усвоения кислорода дрожжами, можно из уравнений (3) и (4) рассчитать также $K_{L a}$ из равенства

$$
K L a=\frac{K r}{C o-C}=\frac{\mu \cdot x \cdot q}{C o-C} .
$$

Разность $C o-C$, характеризующая скорость растворения и потребления кислорода, зависит от концентрации дрожжей, скорости их размножения и потребности в кислороде для синтеза 1 г биомассы. С увеличением х содержание кислорода в среде падает до нуля $(C=0)$, а скорость растворения кислорода достигает максимума $K_{D}=K_{L a} \cdot C o$, но при этом удельная скорость роста дрожжей принимается соответственно уравнению

$$
\mu=\mu \max \cdot \frac{C}{K s+C},
$$

где $K_{s}$ - константа Михаэлиса-Мэнтена, показывающая концентрацию растворенного кислорода, при котором $\mu$ уменьшается и достигает только половины константы роста $\left(\mu=\mu_{\max }: 2\right)$.

Величина $K$ очень мала и, по данным разных авторов, равна $0,05 \ldots 0,10$ мг/л. В отношении величины $C$ также нет единого мнения. В конкретных условиях требуется та или иная остаточная концентрация растворенного кислорода. Доста- 
точное количество воздуха, подаваемое в среду, характеризуется постоянством величины $C$.

Для дрожжей Saharomices cerevisae, выращиваемых на мелассной среде, растворенного кислорода должно быть не менее 0,23 мг/л, т.е. около $3 \%$ от полного насыщения им воды при $30{ }^{\circ} \mathrm{C}$ (7,63 мг/л). Остаточная концентрация менее 0,23 мг/л не обеспечивает нормальный выход дрожжей. Если $C$ выше «критической» концентрации растворенного кислорода в среде (около 0,5 мг/л), размножение дрожжей зависит только от их активности и свойств среды [4].

Поскольку скорость использования дрожжами кислорода не зависит от количества растворенного кислорода при концентрации его в среде более 0,5 мг/л, достаточно поддерживать эту величину постоянной. Увеличение ее не ускоряет растворения кислорода, а следовательно и образования биомассы. Произведение $K_{L a} \cdot(C o-C)$ или $K_{L a}(7,63 \ldots 0,5)$ соответствует максимальному количеству кислорода, которое требуется подать в аппарат в течение часа. Разделив эту величину на $K_{r}$, узнаем критическую концентрацию дрожжей

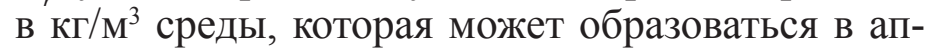
парате при полном обеспечении клеток кислородом, с тем, что остаточное количество его в среде будет поддерживаться на уровне 0,5 мг/л.

В зависимости от скорости разбавления и свойств среды количество растворенного кислорода, определенное полярографом, в аппаратах разной конструкции составило (в мг/л): Фрикса 0,5; Шоллер - Зейдель - Рихе 1,2; Фогельбуша 1,5, с ДИВЭ $2,7$.

Как было показано выше, скорость размножения дрожжей зависит не от концентрации растворенного в жидкости кислорода, а от скорости его растворения, достигающей наибольшей величины, когда $K_{D}=K_{L a} \cdot$ Co.

Но увеличение концентрации дрожжей целесообразно только до определенного предела $\left(x_{\text {кр }}\right)$, пока $K_{D}$ не достигнет указанного предельного значения и пока будет справедливо равенство

$$
x_{k p}=K_{D} /(\mu \cdot q) ; K_{D}=x_{\kappa p} \cdot \mu \cdot q .
$$

При увеличении концентрации дрожжей до критической коэффициент абсорбции кислорода $K_{D}$ также возрастет до предельной величины. Если $x_{\text {кр }}$ в силу флотации клеток в аппарате уве- личивается, $K_{D}$ остается неизменным, но удельная скорость роста $\mu$ падает, производительность аппарата снижается, а расход кислорода на синтез единицы биомассы соответственно увеличивается.

Между скоростью растворения кислорода и количеством затрачиваемой на аэрацию и перемешивание энергии существует прямая зависимость, причем величина $K_{D}$ не зависит от конструкции перемешивающего устройства, а только от количества энергии, передаваемой жидкости [5].

Проведенные исследования и предложенная методика определения скорости растворения кислорода и коэффициента массообмена при культивировании дрожжей расы Saharomices cerevisae с использованием метода ДИВЭ позволяет сделать следующие выводы.

- Дискретно-импульсный ввод энергии интенсифицирует процесс массообмена и растворения кислорода в питательной среде, что увеличивает скорость роста дрожжей на $35 . .45 \%$.

- Скорость роста дрожжей зависит не от концентрации растворённого в питательной среде кислорода, а от скорости его растворения.

- Величина $K_{L a}$ зависит от качества питательной среды, типа массообменного оборудования и гидродинамического режима его работы. Сравнивать эффективность различных массообменных аппаратов можно лишь по значению $K_{L a}$ для каждого из них отдельно, а не по скорости абсорбции, так как последняя тем больше, чем ближе к нулю фактическое содержание кислорода в среде $(C \sim 0)$ и тем меньше, чем ближе к насыщению содержание в ней кислорода $(\mathrm{Co})$.

\section{ЛИТЕРАТУРА}

1. Долинский А.А. Тепломассообмен и гидродинамика в парожидкостных дисперсных средах. Теплофизические основы дискретно - импульсного ввода энергии. / А.А. Долинский, Г.К. Иваницкий. - К.: Наукова думка, 2008. - 381 с.

2. Мальцеев П.М. Технология бродильных производств/ П.М. Мальцев - 2-е изд., перераб. и доп. - М.: Пищевая промышленность, 1980. - 569 c.

3. Коновалов С.А. Биохимия дрожжей./ С.А. 
Коновалов. - М.: Пищепромиздат, 1962. - 272 стр.

4. Карелин Я.А. Очистка производственных сточных вод в аэротенках/ Я.А. Карелин, Д.Д. Жуков, В.Н. Журов, Б.Н. Репин - М.: Стройиздат , $1973-223 \mathrm{c}$.

\section{EXPERIMENTAL DETERMINATION OF THE MASS TRANSFER COEFFICIENT BY CULTURING MICROORGANISMS USING METHOD OF DISCRETE-PULSED ENERGY INPUT}

\author{
Obodovich A.N. ${ }^{1}$, Mudrak T. O. ${ }^{2}$, Kostik S.I. ${ }^{1}$, \\ Sidorenko V.V. ${ }^{1}$
}

${ }^{1}$ Institute of Engineering Thermophysics, National Academy of Sciences of Ukraine, Zhelyabova str., 2a, Kiev, 03680, Ukraine

${ }^{2}$ National University of Food Technologies, Volodymyrskaya str., 68, Kiev, 01601, Ukraine

The paper presents the ways of intensifying the process of cultivation of microorganisms by discrete - pulse energy input into the culture medium in order to strengthen its aeration. Installation presented on the basis of the rotary-pulsation apparatus, allowing to implement this method, described the principle of its operation and optimum operating parameters. On the example of bakery yeast culturing the technique of determining the mass-transfer coefficient is presented.

References 5, fig. 4.
5. Соколенко А.И. Дрожжерастильные аппараты и массообмен при аэрации (обзор) / А.И. Соколенко, М.П. Гандзюк, А.Ц. Мардер - М.: ЦНИИТЭИ Пищепром, 1971 - 22 с.
Key words: aeration, the mass transfer coefficient, rotary-pulsation apparatus, yeast.

1. Dolinskij A.A. Heat and mass transfer and hydrodynamics in the vapor-liquid dispersed media. Thermophysical using discrete - input pulse energy/ A.A. Dolinskij, G.K. Ivanickij - K.: Naukova dumka, $2008-381 \mathrm{p}$.

2. Maltsev P.M. Fermentation Technology 2-d edition / P.M. Maltsev. - M.: Pishevaya promyshlennost, $1980-560 \mathrm{p}$.

3. Konovalov S.A. Biochemistry of yeast /S.A. Konovalov. - M.: Pishepromizdat, 1962. - 272 p.

4. Karelin Ya.A. Process Wastewater Treatment in the aeration tanks /Ya.A. Karelin, D.D. Zhukov, V.N. Zhurov, B.N. Repin - M.: Strojizdat, 1973 223p.

5. Sokolenko A.I. Yeast germinators apparatus and mass transfer with aeration (review) / A.I. Sokolenko, M.P. Gandzyuk, A.C. Marder - M.: CNIITEI Pisheprom, $1971-$ p.22.

Получено 06.04.2015 Received 06.04.2015 